\title{
Inoculación de Consorcios Micorrícicos Arbusculares en Coffea arabica, Variedad Caturra en la Región San Martín
}

\author{
Karen M. Del Aguila ${ }^{1}$, Geomar Vallejos-Torres ${ }^{2}$, Luis A. Arévalo ${ }^{2}$ y Alejandra G. Becerra ${ }^{3}$ \\ (1) Universidad Nacional de San Martín-Tarapoto. Jr. Amorarca 315 - San Martín - Perú. \\ (e-mail: marilu.parillo@outlook.com) \\ (2) Instituto de Investigaciones de la Amazonía Peruana. Jr. Belén Torres de Tello 135, San Martín - Perú. \\ (e-mail: gvallejost@gmail.com; larevalol@yahoo.es) \\ (3) Instituto Multidisciplinario de Biología Vegetal (IMBIV) - CONICET. Universidad Nacional de Córdoba - \\ Argentina. (e-mail: abecerra@unc.edu.ar)
}

Recibido Jun. 1, 2017; Aceptado Ago. 8, 2017; Versión final Oct. 13, 2017, Publicado Feb. 2018

\begin{abstract}
Resumen
El objetivo del presente estudio fue determinar el efecto de nueve consorcios micorrícicos arbusculares y la inoculación a plántulas de café Coffea arabica, variedad Caturra, comparados con un control (sin inoculación), durante siete meses en vivero. El cultivo de café en la Región San Martín es una de las actividades agrícolas económicas más importantes. Una alternativa para incrementar la producción consiste en la aplicación de hongos micorrizas. Se empleó un Diseño Completamente al Azar (DCA), nueve tratamientos y un control constituido por tres repeticiones (6 plantas/repetición). La procedencia de los consorcios micorrícicos son de fincas cafetaleras de la Región San Martín en Perú. Se evaluaron el porcentaje de colonización, longitud de micelio extraradical, altura de planta, área foliar, biomasa aérea y biomasa radicular seca. Los resultados del estudio mostraron que tres de los nueve consorcios estudiados fueron más eficientes en el crecimiento y desarrollo de las plantas de café.
\end{abstract}

\section{Inoculation Effect of Arbuscular Mycorrhizal Consortiums in Coffea arabica, Caturra Variety in the San Martín Region}

\begin{abstract}
The objective of this study was to determine the inoculation effect of nine consortiums of Arbuscular Mycorrhizal Fungi (AMF) in coffee seedlings Coffea arabica, Caturra variety, compared with a control (without inoculation), during seven months under greenhouse conditions. The production of coffee in San Martín-Perú, is one of the most important agricultural economic activities. An alternative to increase its production is the employment of AMF. The experiment was done using a completely randomized design, with nine treatments and three repetitions (6 plants/repetition). The origin of the mycorrhizal consortia came from coffee farms of provinces distributed at San Martín Region. The study was performed evaluating parameters as colonization (\%), extraradical mycelium length, plant height, leaf area, aerial biomass and dry root mass. The results of the study show that three of the nine consortia studied were more efficient during the growth and development of coffee plants seedlings.
\end{abstract}




\section{INTRODUCCIÓN}

El café constituye para el Perú, uno de los productos agrícolas más importante en el comercio internacional (Julca et al., 2013), se desarrolla en 11 regiones e involucra a 67 provincias y 338 distritos rurales. La caficultura peruana, a pesar de su importancia económica, enfrenta limitaciones en cuanto al manejo del cultivo (deficientes prácticas culturales, agotamiento de suelos, difusión de plagas y enfermedades) que conllevan a índices de productividad por debajo de nuestros países vecinos, no permitiendo mejor posicionamiento en el mercado internacional (MINAGRI, 2014). Además existe desconocimiento de la importancia de la actividad microbiológica del suelo. Los Hongos Micorrícicos Arbusculares (HMA), son simbiontes obligados de un amplio rango de especies vegetales. Las simbiosis son biótrofas y normalmente mutualistas, siendo las interacciones compatibles de larga duración basadas principalmente en la transferencia bidireccional de nutrientes entre los simbiontes (Smith y Read, 2008). Una de las características que se ha descubierto en las micorrizas es que éstas se encuentran en forma natural en todos los suelos.

El desarrollo de la simbiosis planta - hongo, induce cambios en la fisiología de la planta que la hace más resistente a diferentes tipos de estrés ambiental, además de tener influencia directa en la estructura del suelo y la multifuncionalidad de los ecosistemas (Smith y Read, 2008). Sin embargo, en Perú hasta ahora no se ha registrado el estudio de la influencia de consorcios micorrícicos sobre el crecimiento y desarrollo de plántulas de café en vivero. Este estudio está orientado a generar conocimiento sobre el efecto de los HMA en la producción de plantas de café vigorosas con características morfológicas ideales para su trasplante a campo definitivo.

\section{MATERIALES Y MÉTODOS}

La metodología se presenta en dos subsecciones donde se detallan el desarrollo del ensayo y las evaluaciones de los parámetros en estudio.

\section{Conducción del experimento}

El estudio se realizó en las instalaciones del Laboratorio de Micorrizas Arbusculares (LMA) y del vivero de Multiplicación de Hongos Micorrícicos Arbusculares (HMA) del Instituto de Investigaciones de la Amazonía Peruana, ubicado en el Departamento de San Martín, Provincia de San Martín, Distrito de Tarapoto, localizado entre las coordenadas $06^{\circ} 28^{\prime}$ latitud sur y $76^{\circ} 22^{\prime}$ longitud oeste, a una altitud de 330 m.s.n.m.m.

\section{Obtención de las fuentes de inóculo}

Se seleccionaron tres provincias cafetaleras importantes en la Región San Martín: Lamas, Moyobamba y El Dorado y dentro de ellas se seleccionaron tres localidades distintas de acuerdo a la altitud y en cada localidad se tomaron muestras de suelo de tres plantas de café como fuentes de inóculo. Se colectaron tres muestras de suelo más raicillas de las plantas de aproximadamente $1 \mathrm{~kg}$ por cada localidad evaluada. La distribución y procedencia de las fuentes de inóculo se presentan en la Tabla 1. Las muestras de suelo se tomaron a profundidad de 0 a $15 \mathrm{~cm}$, separadas a $30 \mathrm{~cm}$ del tallo principal en tres puntos diferentes. Estas fuentes de inóculos colectadas pasaron a las instalaciones del vivero para ser multiplicados en macetas utilizando plantas trampas de maíz Zea mays, por un periodo de 60 días.

\section{Cuantificación de esporas de HMA}

La cuantificación de esporas se realizó según el método de decantado húmedo y tamizado propuesto por Gerdemann y Nicolson (1963), a través de dos tamices de 250 y $38 \mu \mathrm{m}$ respectivamente y centrifugado con solución de sacarosa al 45\% (Sieverding, 1984). Por cada muestra se realizaron tres conteos, para obtener un estimativo del número total de esporas. Luego del periodo de multiplicación de los HMA en las plantas trampas, el sustrato de cada cama se utilizó como fuente de inóculo para los tratamientos testados.

\section{Trasplante de las plántulas de café e inoculación con HMA}

Se preparó una mezcla de suelo agrícola, con arena $(2: 1 \mathrm{v} / \mathrm{v})$, desinfectada por medio del calor generado de la energía solar capturada (solarización), durante 45 días, para eliminar los patógenos del suelo que podrían contaminar los ensayos (Díez-Rojo, 2006). El sustrato homogenizado y desinfectado se colocó en bolsas de polietileno color negro de aprox. $3 \mathrm{~kg}$, tanto el repique como la inoculación con HMA se realizaron simultáneamente para reducir el estrés de las plántulas y poner en contacto directo el inóculo con las raíces. Para ello, las plántulas de café emergidas que ya tenían totalmente abiertas las hojas cotiledonales (fase mariposa), fueron extraídas cuidadosamente de las camas germinadoras y repicadas en cada bolsa almaciguera; todas las bolsas repicadas fueron etiquetadas y codificadas. Las plántulas se inocularon individualmente con 1500 esporas por planta en todos los tratamientos. El ensayo constituyó de 9 tratamientos con inóculos y un tratamiento sin inóculo (Control). 
Tabla 1: Distribución de los tratamientos y procedencia de las fuentes de inóculos de HMA.

\begin{tabular}{|c|l|c|}
\hline No Tratamientos & \multicolumn{1}{|c|}{ Descripción } & Altitud (msnm) \\
\hline T0 & Testigo - Sin HMA. & - \\
\hline T1 & HMA procedencia Lamas - Chirapa. & $800-1000$ \\
\hline T2 & HMA procedencia Lamas - Aviación. & $1000-1200$ \\
\hline T3 & HMA procedencia Lamas - Chontalillo. & $1200-1400$ \\
\hline T4 & HMA procedencia Moyobamba - Calzada. & $800-1000$ \\
\hline T5 & HMA procedencia Moyobamba - Los Ángeles. & $1000-1200$ \\
\hline T6 & HMA procedencia Moyobamba - Potrerillo. & $1200-1400$ \\
\hline T7 & HMA procedencia El Dorado - Nueva Juventud. & $800-1000$ \\
\hline T8 & HMA procedencia El Dorado - Buena Vista. & $1000-1200$ \\
\hline T9 & HMA procedencia El Dorado - Monte de los Olivos. & $1200-1400$ \\
\hline
\end{tabular}

\section{Evaluaciones}

Siete meses después de la siembra e inoculación de HMA, seis plántulas de café por repetición fueron evaluadas con los siguientes índices:

Altura de planta: La medición se realizó desde la base del tallo hasta el ápice de la planta, utilizando una regla milimetrada de $30 \mathrm{~cm}$, se utilizó la misma regla para todas las evaluaciones. El material de medición fue desinfectado con alcohol al 96\%, para evitar la contaminación entre tratamientos.

Biomasa seca de la parte aérea y radicular: Para esta evaluación fueron seleccionadas cuatro plantas aleatoriamente por cada tratamiento. La biomasa se obtuvo mediante el peso de los componentes de las plantas (raíz, tallo y lámina foliar) en una báscula analítica después de haberse secado en una estufa de aire forzado a $60^{\circ} \mathrm{C}$ por $48 \mathrm{~h}$.

Área Foliar (AF): Para la determinación del área foliar se tomaron fotografías a las hojas de las plantas de café. El cálculo se hizo mediante el uso del software ASSES, que permite calcular el área foliar usando colores de contraste y la calibración de un objeto de área conocida.

Determinación del porcentaje de colonización micorrícica: Se realizó en raíces jóvenes (secundarias y terciarias) del café, siguiendo la técnica descrita por Phillips y Hayman (1970) y modificada por Kormanik et al., (1980) en su trabajo Procedures and equipment for staining large numbers of plant root samples for endomycorrhizal assay citado por Kormanik et al., (1982), también conocido como método de tinción de raíces.

Estimación del grado de colonización micorrícica: Se calculó utilizando la metodología de Trouvelot et al., (1986), para ello cada muestra estuvo conformada por 30 fragmentos de raíz teñida de $1 \mathrm{~cm}$ de longitud, colocadas verticalmente en paralelo una a continuación de otra sobre una lámina portaobjetos y montadas con una gota de lactoglicerol. Luego de cubrir las raíces con láminas cubreobjetos, éstas se observaron en estereomicroscopio con aumentos de 10x y 40x. Finalmente, para el cálculo de la intensidad de la colonización en el sistema radicular se utilizó la siguiente fórmula: $(95 n 5+70 n 4+30 n 3+5 n 2+n 1) /$ Total de raíces montadas.

Dónde: $\mathrm{n} 5$ = número de fragmentos clasificados como $5 ; \mathrm{n} 4$ = número de fragmentos clasificados como 4 ; n3 = número de fragmentos clasificados 3, n2 = número de fragmentos clasificados como 2 y n1 = número de fragmentos clasificados como 1.

Determinación de la longitud de micelio extraradical (LMER): La tinción de micelio extraradical se realizó utilizando la técnica del gel semisólido y cuantificación por el método de intersección de cuadrantes (Carballar, 2009), de acuerdo con el siguiente procedimiento: Para la estimación de la longitud de micelio extraradical las muestras preparadas se observaron en estereomicroscopio con aumentos de $5 x$ y 10x, contándose las intersecciones hifa-línea. La cantidad numérica obtenida se trasformó a longitud de micelio por unidad de peso de suelo utilizando la fórmula de Newman (1966). 


\section{Diseño estadístico y análisis de datos}

Se utilizó el diseño completamente al azar (DCA), con 9 tratamientos y un control, con nivel de significancia de $\alpha=0,05$. Los datos de biomasa seca fueron transformados a $\sqrt{ } x+1$ (Padrón, 1996); mientras que para los datos de la variable porcentaje de colonización se utilizó la transformación angular arcsen $\sqrt{x} \%$ (Box y Hunter, 1989). Cada tratamiento estuvo constituido por 18 unidades experimentales y cada unidad experimental fue representada por una planta de café variedad Caturra. Con los datos obtenidos se realizó el análisis de variancia y para la comparación de las medias se utilizó la prueba de Tukey con nivel de significancia $\alpha=0,05$.

\section{RESULTADOS Y DISCUSIÓN}

Los hongos micorrízicos arbusculares (HMA), colonizan dos tipos de ambientes, el primero ocurre en las raíces de la planta hospedera y el segundo ocurre en el suelo circundante, ambos a través de sus hifas (Jansa, Bukovská y Gryndler, 2013). La variable colonización determinó el porcentaje de ocupación de los HMA nativos en las raíces de café (Coffea arabica L.), el cual fue de un total de $31,33 \pm 11,50 \%$ (Fig. 1). Así mismo T1 presentó un valor inferior de colonización que varió de 11,50 a 15,83\% en comparación con los demás tratamientos de 24,17 a $31,33 \%$.

Entre los tratamientos inoculados no presentaron diferencias estadísticas significativas entre las medias en concordancia con la prueba de Tukey $(P<0,05)$. Esta diferencia podría ser explicada por la utilización de diferentes consorcios micorrícicos y su diversidad de especies, que podrían diferir en el comportamiento de su colonización, tanto en el tiempo de establecimiento de la simbiosis, así como las condiciones particulares del suelo que permiten la simbiosis (Hart y Reader, 2002; Zangaro et al., 2012.)

Por otro lado, la variable Longitud de micelio extraradicular (LMER), determinó la presencia de los HMA nativos en el suelo circundante a las raíces, donde ocurre la absorción de nutrientes, razón por la cual su estudio es reconocido como un aspecto importante de la simbiosis (Helgason y Fitter, 2009), en este trabajo los resultados obtenidos con la inoculación de HMA, difieren con el control, lo cual indica que los consorcios micorrícicos fueron capaces de establecer la simbiosis conocida como micorriza con las plantas de café.

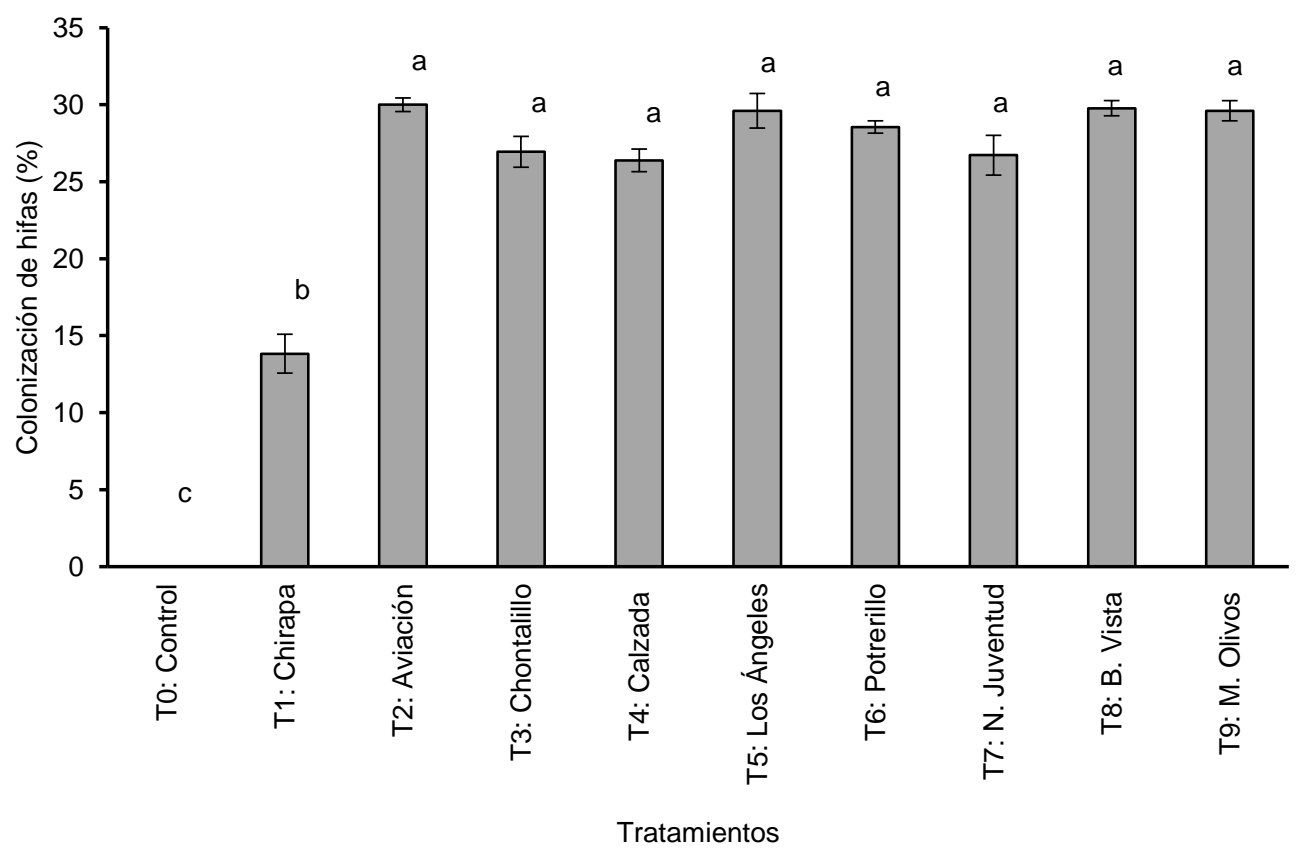

Fig. 1: Porcentaje de colonización en las raíces de café

La variable longitud de micelio extraradical se muestra en la Fig. 2. Se puede observar que T9, obtuvo el mayor valor para esta variable con diferencias significativas sobre los demás que varió de 120 a $149 \mathrm{~cm}$, seguidos por T5, y T8, sin diferencias estadísticas significativas entre ellos. T4, y T6, fueron los que obtuvieron los menores valores de 40 a $50 \mathrm{~cm}$, con diferencias significativas entre ellos. Sin embargo aún éstos fueron mayores que T0, que no presentó colonización, como consecuencia de no haber sido inoculado con HMA nativos del cultivo de café. 
Se observa que la longitud de micelio extraradical de los tratamientos inoculados con HMA, sin importar su procedencia, presentó diferencias estadísticas significativas en comparación con T0. Es importante mencionar que T1, que presentó un bajo porcentaje de colonización micorrícica, en cuanto a la variable longitud de micelio extraradical (LMER), superó a T3, T4, y T6, lo cual explica que la LMER no depende del grado de colonización sino de la especie de HMA (Carballar, 2009).

A diferencia de la colonización, con esta variable se ha corroborado lo mencionado por López, (2012), quien mencionó que es mediante el micelio extraradical que se lleva a cabo la función nutricional, logrando de esta manera influir favorablemente en el crecimiento y desarrollo de las plantas. Con los resultados obtenidos se constató que el micelio externo funcionó como una extensión física del sistema radicular (Finlay, 2008), logrando alcanzar $148,72 \mathrm{~cm}$ para T9.

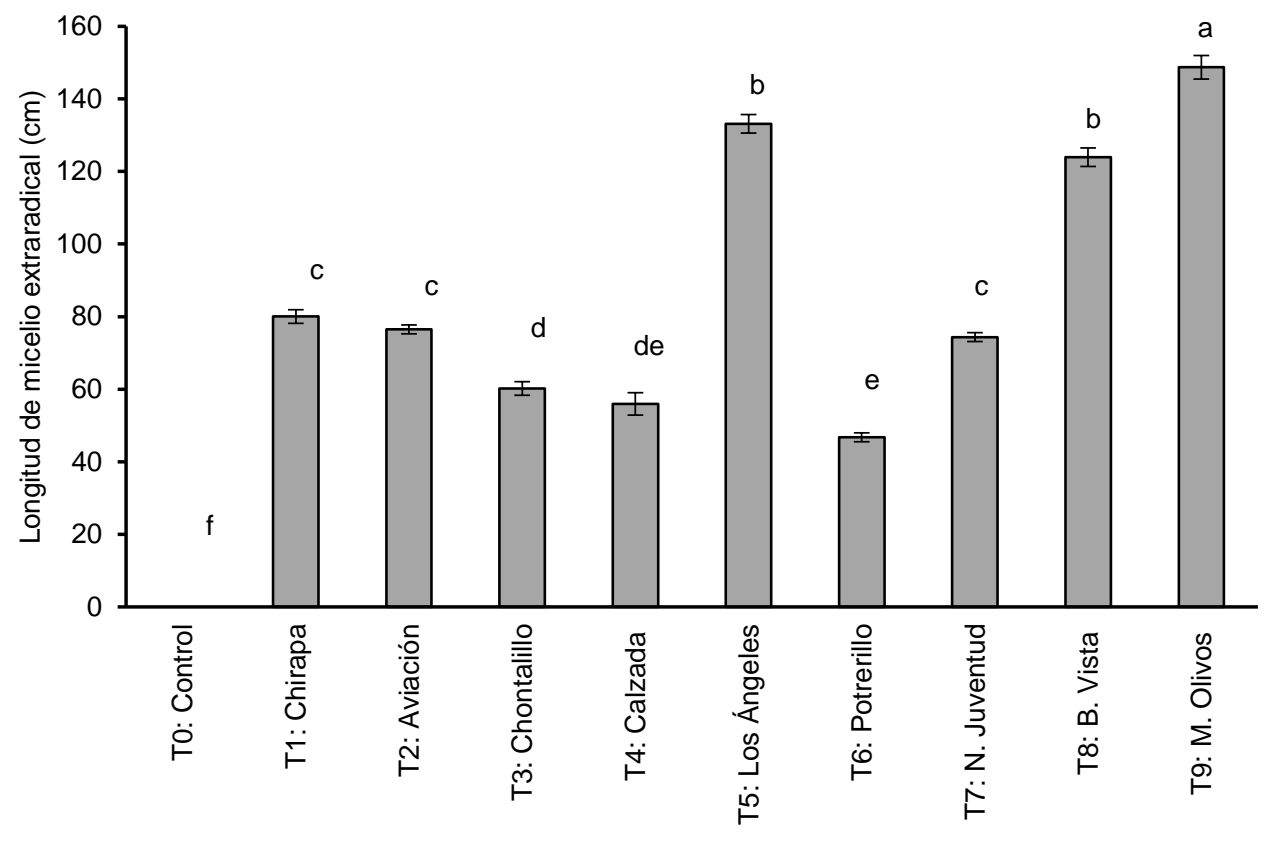

Tratamientos

Fig. 2: Longitud de micelio extraradical (LMER), inoculadas con hongos micorrízicos arbusculares

Las plantas de café inoculadas con micorrizas presentaron mejores características anatómicas después de 7 meses de establecidas en condiciones de vivero. El análisis de varianza de los datos de altura de las plantas, indicó que existe diferencia significativa entre los tratamientos inoculados comparados con T0, siendo T8, el mejor para la variable altura de planta. Las plantas fueron en promedio $10,65 \%$ más altas que el control (Fig. 3). Esto pudo deberse a que las plantas de café variedad Caturra son "Altamente dependientes" a la asociación micorrizal (Jaramillo y Osorio, 2009), y en consecuencia éstas pudieron beneficiarse de la influencia que los HMA ejercen en el crecimiento de las plantas (Osorio, 2012).

Por tanto se puede indicar que las diferencias estadísticas observadas en los tratamientos inoculados con HMA, pudieron haber sido causadas por la diversidad de especies que presentaron los consorcios micorrícicos utilizados como fuente de inóculo.

Resultados semejantes fueron observados por Trejo et al., (2011), en un estudio conducido bajo condiciones controladas de invernadero, aplicaron consorcios micorrícicos arbusculares que incrementaron la altura de las plántulas del café en $91 \%$ con respecto a plantas no inoculadas. El mejor momento para establecer la simbiosis entre los HMA y las plantas de café es su estado vegetativo inicial; puesto que significa un mejor aprovechamiento de nutrientes, un ahorro en fertilizantes químicos y una manera de asegurar el mayor número de plantas vigorosas para el trasplante a campo definitivo (Alarcón y FerreraCerrato, 1999).

Los incrementos del área foliar en las plantas inoculadas oscilaron desde 77,45 hasta $239,31 \%$, encontrándose efectos positivos en todos los tratamientos inoculados comparados con T0, corroborando la alta dependencia micorrícica del café (Jaramillo y Osorio, 2009). Resultados semejantes encontró Fernández-Martín et al., (2005), en sus investigaciones realizadas en café variedad Catuaí en etapa de vivero, con incrementos entre 6 y $140 \%$ de área foliar con respecto a plantas no inoculadas, resultados similares se obtuvieron con el ensayo según la Fig. 4. 


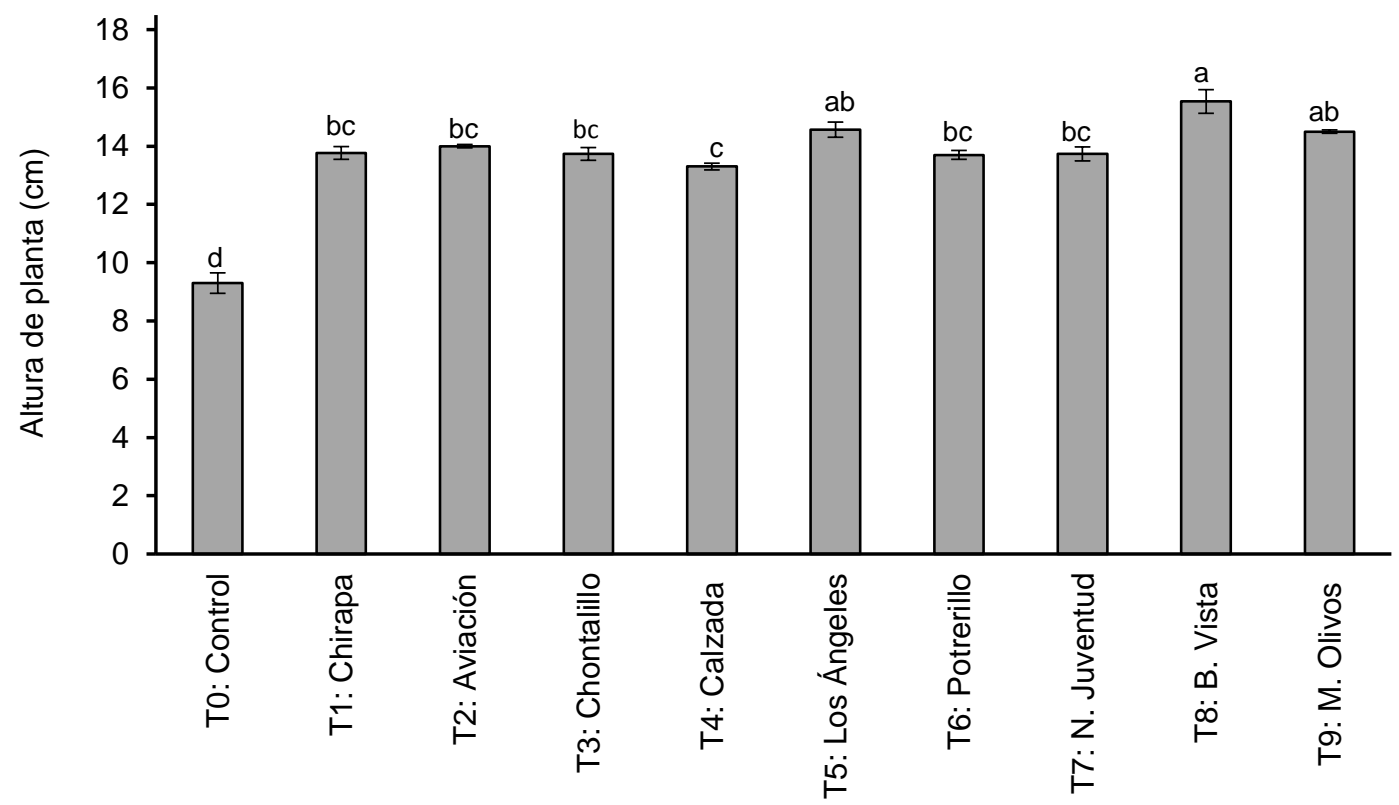

Tratamientos

Fig. 3: Altura de planta $(\mathrm{cm})$ de café inoculadas con hongos micorrízicos arbusculares

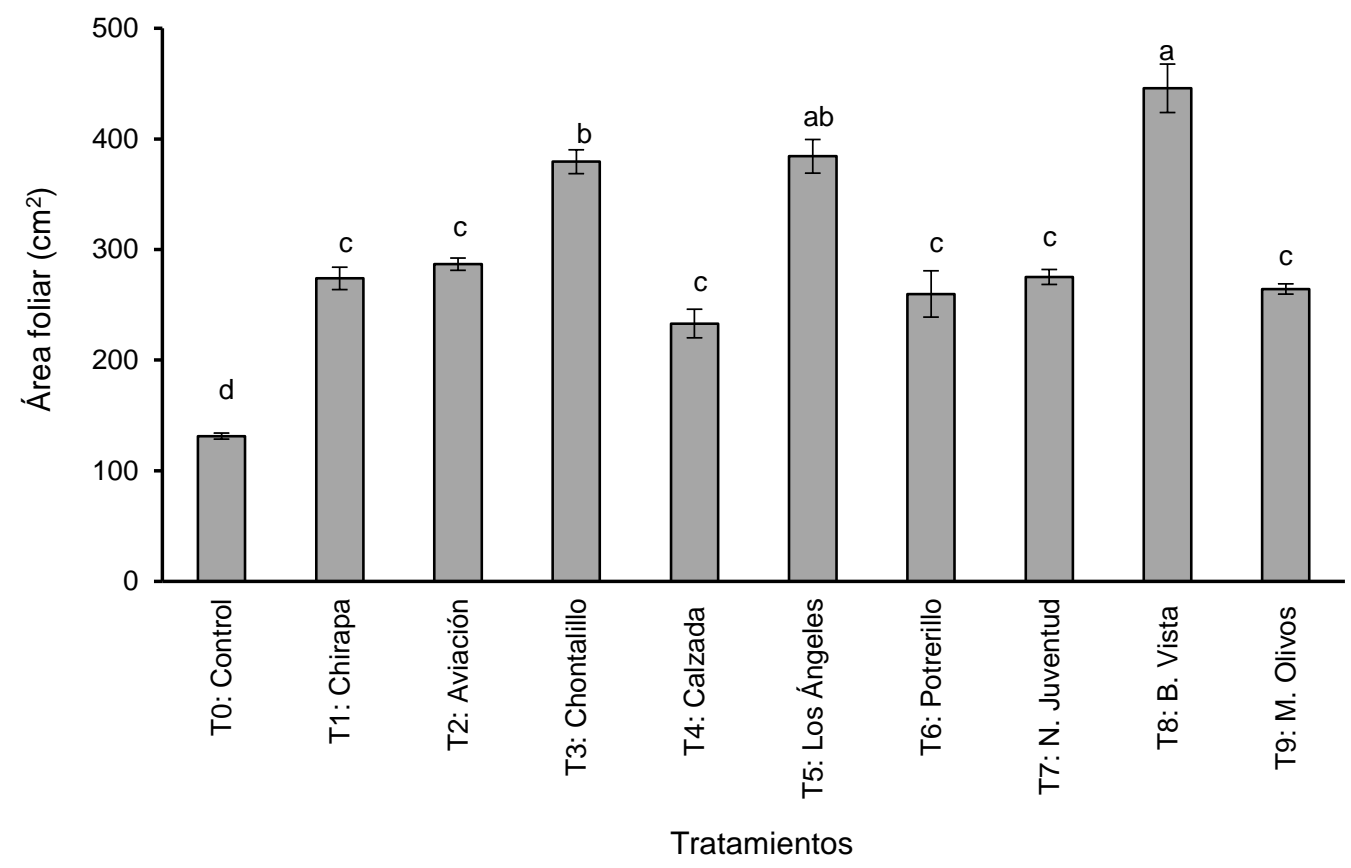

Fig. 4: Área foliar $\left(\mathrm{cm}^{2}\right)$ de plántulas de café inoculadas con hongos micorrízicos arbusculares

Al igual que el crecimiento de la planta y el área foliar, la producción de biomasa seca es considerada como un indicador de la evidencia de los beneficios que una planta hospedadora de HMA puede obtener de la simbiosis micorrícica. En los resultados se observaron incrementos de la biomasa seca área de los tratamientos inoculados con HMA, que fluctuaron entre 85,51 y $201,64 \%$ más que las plantas no inoculadas (Fig. 5). Para esta variable, los mejores resultados se obtuvieron con los tratamientos T5, T8 y T9, procedentes de las provincias de Moyobamba y El Dorado.

Un trabajo realizado por Cuervo, (2017) quien inoculó plantas de Coffea arabica con Rhizoglomus fasciculatum (anteriormente llamado Glomus fasciculatum), obtuvo resultados significativamente superiores de biomasa seca aérea en dichas plantas comparadas con aquellas que no fueron inoculadas con el hongo micorrícico. 
En la Fig. 6 se observan incrementos de la biomasa seca radicular para todos tratamientos inoculados con HMA que fluctuaron entre 77,16 y $203,22 \%$, en comparación con las plantas no inoculadas. Diversos estudios en condiciones de invernadero demuestran que la asociación simbiótica produce diversos cambios y/o modificaciones a nivel fisiológico y morfológico destacándose los incrementos en la actividad fotosintética por efecto de la mayor capacidad de fijación de $\mathrm{CO}_{2}$, incrementando la tasa de crecimiento y biomasa. Los resultados corroboran lo mencionado por Al-Areqi et al., (2014), quienes afirmaron que las plantas de Coffea arabica inoculadas con HMA en su estado vegetativo inicial obtienen mayor cantidad de biomasa radicular.

En este estudio la inoculación con los consorcios micorrícicos arbusculares nativos presentaron una ventaja respecto a la obtención de plantones aptos para el trasplante a campo definitivo.

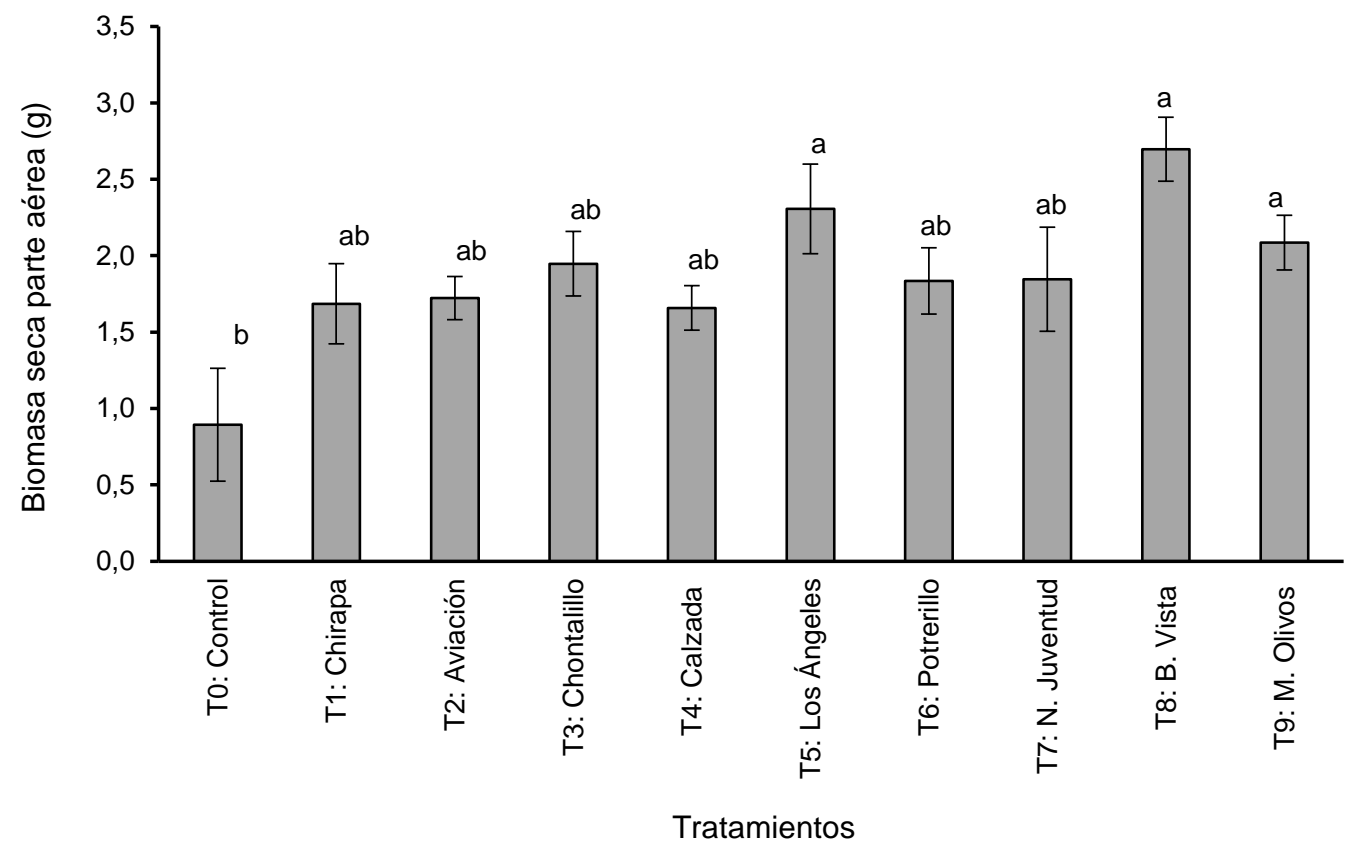

Fig. 5: La biomasa seca de la parte aérea $(\mathrm{g})$ inoculadas con hongos micorrízicos arbusculares

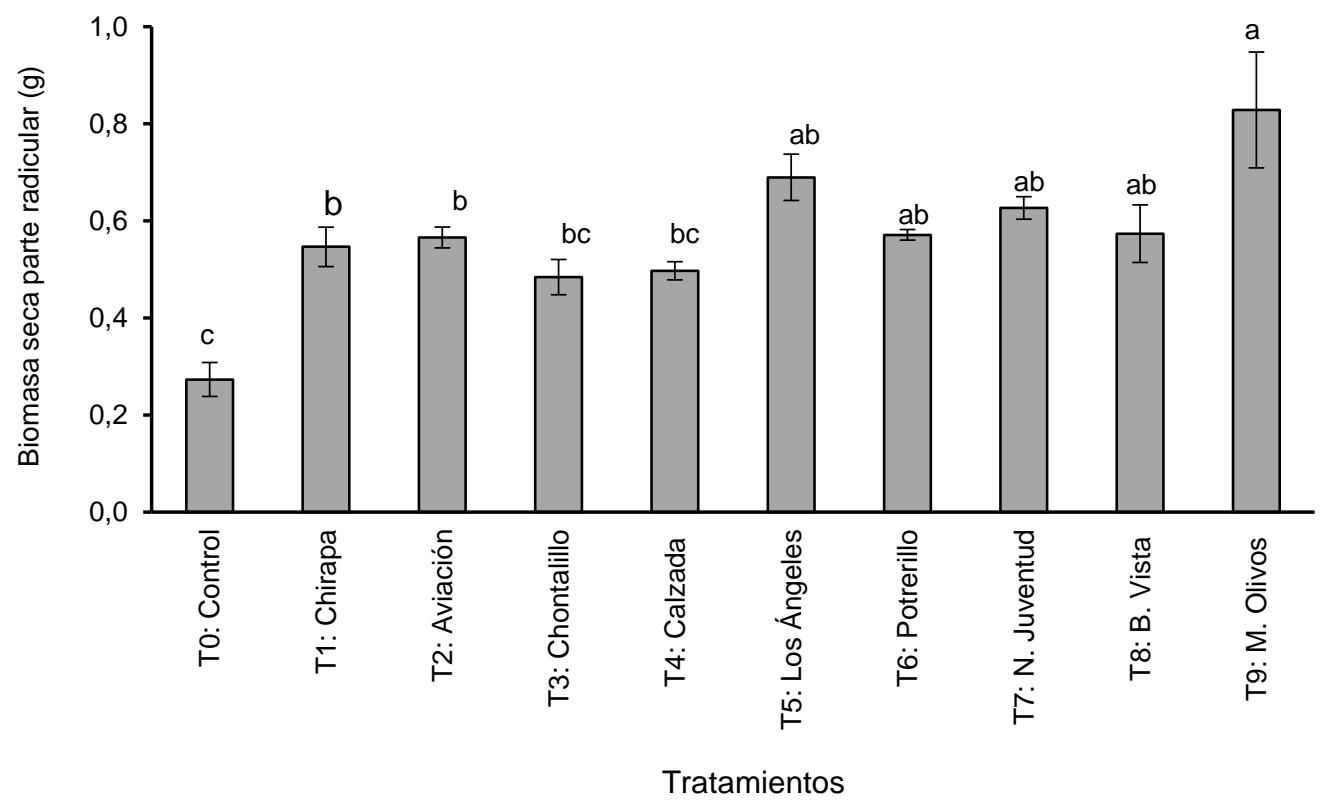

Fig. 6: La biomasa seca radicular (g) en plántulas de café inoculadas con hongos micorrízicos arbusculares

Se encontró que existió una fuerte correlación entre longitud de micelio extraradical (LMER), con la altura de planta y biomasa radicular seca en todos los consorcios estudiados, con altos y significativos coeficientes de determinación ( $\mathrm{R}^{2}$ : 0,72 y 0,73 respectivamente). No obstante las variables área foliar y biomasa área seca presentaron una moderada correlación entre la longitud de micelio extraradical $\left(R^{2}: 0,60\right.$ y 0,62 respectivamente) (Tabla 2$)$. 
Este resultado nos sugiere que la longitud de micelio extraradical ha influenciado en el crecimiento y desarrollo de las plantas de café y que los consorcios de HMA, han sido efectivos en todos los tratamientos inoculados comparados con el control. Estas correlaciones se explicarían por los cambios de orden fisiológico y morfológico ocurridos en las plantas inoculadas a causa de la simbiosis con los HMA, luego de la colonización y desarrollo del micelio extraradical (Zangaro et al., 2012), fueron las que lograron incorporar los nutrientes necesarios para incrementar las tasas de crecimiento y desarrollo significativamente mayores en comparación con las plantas no inoculadas. Dichos incrementos se vieron reflejados en cada una de las variables morfológicas evaluadas en el presente estudio.

Tabla 2: Análisis de correlación lineal de Pearson entre la longitud de micelio extraradical y las variables morfológicas evaluadas de las plantas de café.

\begin{tabular}{|l|c|}
\hline \multicolumn{1}{|c|}{ Parámetros } & $\begin{array}{c}\text { Longitud de micelio } \\
\text { extraradical }\end{array}$ \\
\hline Altura de planta & 0,72 \\
\hline Área foliar & 0,60 \\
\hline Biomasa aérea seca & 0,62 \\
\hline Biomasa radicular seca & 0,73 \\
\hline
\end{tabular}

\section{CONCLUSIONES}

El estudio ha demostrado que la inoculación de HMA-N en plántulas de café variedad Caturra, presenta efectos positivos sobre los diferentes parámetros morfológicos de la planta, estableciéndose que los mejores consorcios micorrízicos fueron los provenientes de las provincias de Moyobamba y El Dorado (T5, T8 y T9).

\section{AGRADECIMIENTOS}

Al fondo Nacional de Ciencia y Tecnología- FONDECYT por financiar el presente trabajo de investigación en el marco de las actividades del proyecto "Biorestauración de suelos con Hongos Micorrízicos nativos en fincas con café arábicos (Coffea arabica L.), en la Amazonía Peruana, a los ingenieros Luis Alberto Arévalo López investigador principal del proyecto, Geomar Vallejos Torres y Mike A. Corazon Guivin.

\section{REFERENCIAS}

Alarcón, A. y R. Ferrera-Cerrato, Manejo de la micorriza arbuscular en sistemas de propagación de plantas frutícolas, Montecillo, Estado de México. Terra, 17(3), 179- 191 (1999)

Al-Areqi, A. H., M. Chliyeh., A. Ouazzani, R. Benkirane y A. Douira, Effect of a composite endomycorrhizal inoculum on the growth of Coffea arabica seedlings. International journal of plant, animal and environmental sciences, 4(1), 185-194 (2014)

Box, G. y W. Hunter, Estadística para investigadores. Introducción al diseño de experimentos, análisis de los datos y construcción de modelos. Ed. Reverté S.A., 675, Barcelona, España (1989)

Carballar, S., Variación temporal de la diversidad de hongos de micorriza arbuscular y el potencial micorrízico en especies silvestres de Agave en Oaxaca, Tesis de Magister, Dpto. Conservación y Aprovechamiento de Recursos Naturales, Instituto Politécnico Nacional (IPN), Oaxaca, México (2009)

Cuervo, J., Dependencia micorrizal de variedades de café bajo diferentes concentraciones de fosfato soluble en el suelo, Tesis de Magister, Dpto. Geomorfología y Suelos, Universidad Nacional de Colombia, Medellín - Colombia (2017)

Díez-Rojo, M.A., Fundamentos fitotécnicos para la aplicación de subproductos agrarios en la mejora de suelos cultivados, Tesis de titulación, Universidad Politécnica de Madrid (2006)

Fernández-Martín, F., R. A. Rivera-Espinosa., A. Hernández-Jiménez, R. A. Herrera-Peraza, K. FernándezSuárez, Inoculación de hongos micorrízicos arbusculares y diferentes relaciones suelo: humus de lombriz sobre el crecimiento del cafeto (Coffea arabica L.) cv. Catuaí bajo la etapa de vivero. Revista Chapingo Serie Horticultura-México, 11(1), 175-184 (2005) 
Finlay, R. D., Ecological aspects of mycorrhizal simbiosis: with special emphasis on the functional diversity of interactions involving the extraradical mycelium. Journal of Experimental Botany, 59(5), 1115-1126 (2008)

Gerdemann, J.W. y T.H. Nicolson, Spores of mycorrhizal Endogone species extracted from soil by wet sieving and decanting. Trans. Brit. Mycol. Soc. 46(2), 235-244 (1963)

Hart, M.M. y R.J. Reader, Taxonomic basic for variation in the colonization strategy of arbuscular mycorrhizal fungi. New Phytologist, 153(2), 335-334 (2002)

Helgason, T. y A.H. Fitter, Natural selection and the evolutionary ecology of the arbuscular mycorrhizal fungi (Phylum Glomeromycota). Journal of Experimental Botany, 60(9), 2465-2480 (2009)

Jansa, J., P. Bukovská y M. Gryndler, Mycorrhizal hyphae as ecological niche for highly specialized hypersymbionts - or just soil free-riders?, Plant Science, 4(134), 1-8 (2013)

Jaramillo, S. P. y N. W. Osorio, Mycorrhizal dependency of coffee seedling at different levels of soil solution phosphorus. Suelos Ecuatoriales, 39(1), 100-106 (2009)

Julca, O. A., A. C. Echevarría., M. Y. Ladera., V. R. Borjas., J. R. Cruz., A. S. Bello y C. R. Crespo, Una revisión sobre la roya del café (Hemileia vastatrix) algunas experiencias y recomendaciones para el Perú, Univ. Nacional Agraria La Molina e Instituto Regional de Desarrollo de Selva. Chanchamayo-Perú (2013)

Kormanik, P.P., R.C. Schultz y W.C. Bryan, The influence of Vesicular-Arbuscular Mycorrhizae on the growth and development of eight hardwood tree species. Forest Science, 28(3), 531-539 (1982)

López, L., Producción de raíces finas y micorrización en café (Coffea arabica L.) cultivado bajo sistema convencional y orgánico en Turrialba, Costa Rica, Tesis de Licenciatura, Dpto. Recursos Naturales y Ambiente, Universidad Nacional de Agricultura, Turrialba - Costa Rica (2012)

MINAGRI, Ministerio de Agricultura y Riego, Dirección General de Competitividad Agraria, "Plan Nacional de Renovación de Cafetales" (2014)

Newman, E.I., A method of estimating the total length of root in a sample. Journal of Applied Ecology, 3(1), 139-145 (1966)

Osorio, N. W., Uso de hongos formadores de micorriza como alternativa biotecnológica para promover la nutrición y el crecimiento de plántulas (en línea: http://www.walterosorio.net, acceso: 12 de mayo 2016), Universidad Nacional de Colombia-Sede Medellín (2012)

Padrón, E., Diseños Experimentales con Aplicaciones a la Agricultura y la Ganadería. Editorial Trillas, México (1996)

Phillips, J. y D. Hayman, Improved procedures for clearing roots and vesicular-arbuscular fungi for rapid assessment of the infection. Transactions of the British (1970)

Sieverding, E., Aspectos básicos de la investigación de la micorriza vesículo arbuscular. Primer Curso Nacional sobre Micorrizas. Facultad de Ciencias Agropecuarias - Palmira. Colombia, Memorias, 86-87 (1984)

Smith, S. y D. Read, Mycorrhizal Symbiosis, $3^{\text {th }}$ Ed. Elsevier Edition, Londres, Inglaterra (2008)

Trejo, D., R. Ferrera-Cerrato., R. García., L. Varela., L. Lara y A. Alarcón, Efectividad de siete consorcios nativos de hongos micorrízicos arbusculares en plantas de café en condiciones de invernadero y campo. Chile. Revista Chilena de Historia Natural, 84(1), 23-31 (2011)

Trouvelot, A., J.L. Kough y V. Gianinazzi-Pearson, Mesure du taux de mycorrhization VA d'un système radiculaire. Recherche de methods d'estimation ayantune signification function elle. En: "Physiological and genetical aspects of mycorrhizae". Gianinazzi-Pearson, V. and Gianinazzi, S. Eds., INRA, Paris, 101-109 (1986)

Zangaro, W., R. A. Alves., L. E. Lescano., A. P. Insanely y M. A. Nogueira, Investment in fine roots and arbuscular mycorrhizal fungi decrease during succession in three Brazilian ecosystems. Biotropica 44(2), $141-150(2012)$ 
